\title{
Deep Auto-Encoders with Sequential Learning for Multimodal Dimensional Emotion Recognition
}

\author{
Dung Nguyen*, Duc Thanh Nguyen*, Rui Zeng, Thanh Thi Nguyen, Son N. Tran, Thin Nguyen, \\ Sridha Sridharan, Life Senior Member, IEEE, and Clinton Fookes, Senior Member, IEEE
}

\begin{abstract}
Multimodal dimensional emotion recognition has drawn a great attention from the affective computing community and numerous schemes have been extensively investigated, making a significant progress in this area. However, several questions still remain unanswered for most of existing approaches including: (i) how to simultaneously learn compact yet representative features from multimodal data, (ii) how to effectively capture complementary features from multimodal streams, and (iii) how to perform all the tasks in an end-to-end manner. To address these challenges, in this paper, we propose a novel deep neural network architecture consisting of a two-stream auto-encoder and a long short term memory for effectively integrating visual and audio signal streams for emotion recognition. To validate the robustness of our proposed architecture, we carry out extensive experiments on the multimodal emotion in the wild dataset: RECOLA. Experimental results show that the proposed method achieves state-of-the-art recognition performance.
\end{abstract}

Index Terms-Multimodal emotion recognition, dimensional emotion recognition, auto-encoder, long short term memory.

\section{INTRODUCTION}

$\mathbf{E}$ MOTION recognition has become a core research field at the intersection of human communication and artificial intelligence. This research problem is challenging owing to emotions of human beings can be expressed in different forms such as visual, acoustic, and linguistic structures [1].

As shown in the literature, there are two main conceptualisations of emotions: categorical and dimensional conceptualisation. Categorical approach defines a small set of basic emotions (e.g., happiness, sadness, anger, surprise, fear, and disgust) relying on cross-culture studies that show humans perceive certain basic emotions in similar ways regardless of their culture [2]. Alternatively, dimensional approach represents emotions into a multidimensional space where each dimension captures a fundamental property of the emotions (e.g., appraising human emotional states, behaviours and reactions displayed in real-world settings). These fundamental properties can be accomplished using continuous dimensions in the "Circumplex Model of Affects" (CMA) [3] including

D. Nguyen is with the School of Information Technology, Deakin University, and with the Faculty of Information Technology, Monash University, Australia.

D. T. Nguyen, and T. T. Nguyen are with the School of Information Technology, Deakin University, Australia.

R. Zeng is with the University of Sydney, Australia.

S. N. Tran is with the University of Tasmania, Australia.

T. Nguyen is with the Applied Artificial Intelligence Institute, Deakin University, Australia.

S. Sridharan and C. Fookes are with the Speech, Audio, Image and Video Technology research lab, Queensland University of Technology, Australia.

*Co-first author

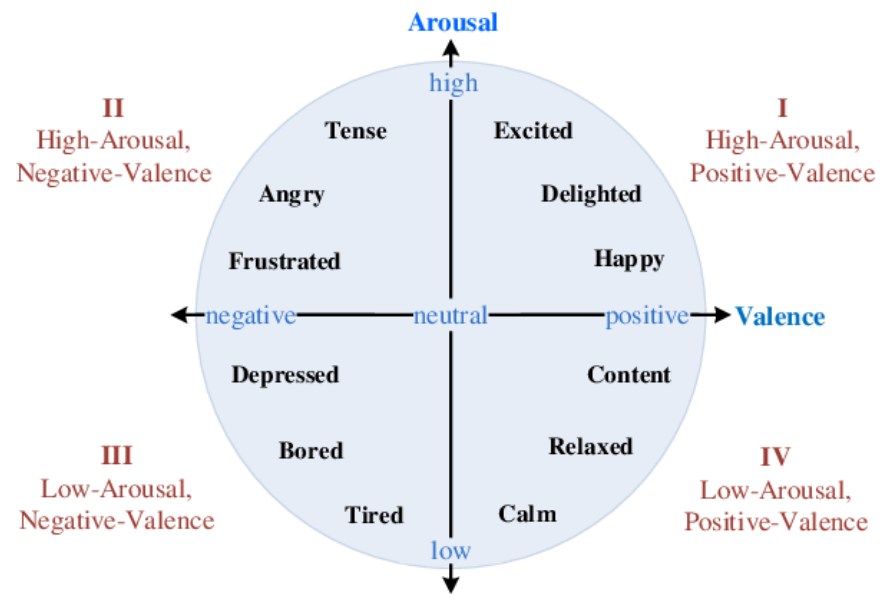

Fig. 1. Two dimensional valence and arousal space (from https://www. pinterest.com.au/pin/354588170647498721//

valence (i.e., how positive or negative an emotion is) and arousal (i.e., the power of the activation of an emotion). Fig. 1 illustrates the CMA. However, this approach is more appropriate to represent subtle changes in emotions, which may not always happen in real-world conditions [2].

Recently, deep neural networks have been proposed to effectively predict the continuous dimensions of emotions based on multimodal cues such as auditory and visual information [2]-[8]. These works combine convolutional and recurrent neural networks for feature integration, taking advantages of automatic feature learning in convolutional networks, while encoding temporal dynamics via the sequential layers in recurrent networks [3]. However, feature integration is performed simply by concatenating domain-dependent features extracted from individual modalities. This scheme is straightforward and simple to implement yet may not be able to effectively learn compact and representative multimodal features. To address this issue, we propose a novel deep neural network for multimodal dimensional emotion recognition using a two-stream auto-encoder incorporating with a long short term memory to perform joint learning temporal and compact-representative features in multimodal data. Our architecture can be end-toend trainable and capable of learning multimodal representations, achieving state-of-the-art performance on benchmark dataset. Specifically, we make the following contributions,

- A two-stream auto-encoder that is able to effectively learn multimodal features from multimodal data for emotion recognition. The constituted auto-encoders ensure 
compact-representative features to be learnt from individual domains while joint training the auto-encoders captures complementary features from the multiple domains. Moreover, auto-encoders are well-known for their robustness to background noise, which is inevitable to auditory signal in practice.

- A long short term memory that enables sequential learning to encode the long-range contextual and temporal information of multimodal features from data streams.

- Extensive experiments on the multimodal emotion in the wild dataset: RECOLA. In our experiments, ablation studies on various aspects of the proposed architecture are conducted. In addition, important baselines including dimensional facial emotion recognition and dimensional speech emotion recognition, and other existing methods are thoroughly evaluated and analysed.

The remainder of this paper is organised as follows: Section III briefly reviews related work; Section III describes our proposed method and its related aspects such as data preprocessing, network architecture, training; Section IV]presents experiments and results; and Section $\mathrm{V}$ concludes our paper with remarks.

\section{RELATED WORK}

Emotion recognition has been a well-studied research problem for several decades and numerous approaches have been proposed. In this section, we limit our review to recent multimodal dimensional emotion recognition methods using deep learning techniques such as convolutional neural networks and long short term memory due to their proven robustness and effectiveness in many applications.

\section{A. Multimodal emotion recognition}

Inspired by the capability of automatic feature learning in deep learning frameworks, Zhang et al. [9] proposed a hybrid deep learning system constructed by a convolutional neural network (CNN), a three-dimensional CNN (3DCNN), and a deep belief network (DBN) to learn audio-visual features for emotion recognition. In this work, the $\mathrm{CNN}$ was pre-trained on the large-scale ImageNet database [10] and used to learn audio features from speech signals. To capture the temporal information from video data, the 3DCNN model in [11] was adapted and fine-tuned on contiguous video frames. The learnt audio and visual features were subsequently fused into the DBN to generate audio-visual features that were finally fed to a linear SVM for emotion recognition.

To classify spontaneous multimodal emotional expressions, Barros et al. [12] proposed a so-called cross channel convolutional neural network (CC-CNN) to learn generic and specific features of emotions based on body movements and facial expressions. These features were further passed into crossconvolution channels to build cross-modal representations. Motivated by human perception in emotional expression, Barros and Wermter [13] developed a perception representation model capturing the correlation between different modalities. In this work, auditory and visual stimuli were firstly fused using the CC-CNN originally introduced in [12] to achieve a multimodal perception representation. A self-organising layer was then applied on top of the CC-CNN to further separate the perceived expression representations.

Li et al. [14] developed a so-called deep fusion convolution neural network (DF-CNN) for learning subject-independent facial features in $2 \mathrm{D}$ and $3 \mathrm{D}$ domains. The DF-CNN is consisted of a feature extraction subnet, a feature fusion subnet, and a softmax layer. For each textured 3D face scan, the DF-CNN represents the face scan via six types of 2D facial attribute maps, which are subsequently passed through the feature fusion subnet to extract and fuse features from both 2D and 3D domains. Emotional expression is predicted by the softmax layer from six dimensional expression probabilities.

Zixing et al. [15] exploited the difficulty in the learning process to improve the prediction phase. In particular, the authors proposed a so-called Dynamic Difficulty Awareness Training (DDAT) procedure taking into account the reconstruction error of input data and annotation uncertainty to estimate the difficulty in learning specific emotions. The estimated difficulty level was subsequently used along with original features to improve the prediction phase. A late fusion was finally conducted to fuse emotion predictions from different modalities. By using the DDAT procedure, the emotion prediction model is improved to better deal with highly difficult regions in the learning process.

Recently, Zheng et al. [16] proposed a multimodal framework including two Restricted Boltzmann Machines (RBMs) to capture eye movements and EEG signal. The RBMs were unfolded into a bimodal deep auto-encoder (BDAE) [17] to extract shared representations of the two modalities, which were finally fed to a linear SVM for emotion classification.

To generate discriminative representations for human emotions, Wei et al. [18] proposed a novel CNN architecture that learns and incorporates the style information extracted from lower layers with content information extracted from high layers. However, instead of directly using emotion labels in the loss function, the authors revised the loss to include the quality of emotion labelling.

Conventionally, emotion recognition approaches classify person-independent emotions directly from observed data or determine the decreasing/increasing intensity of persondependent emotions relatively by comparing video segments. However, Liang et al. [1] proposed to combine both approaches for emotion recognition from audio-visual data. In this work, the emotion recognition task was divided into three subtasks including local relative emotion intensities ranking, global relative emotion intensities ranking, and incorporation of emotion predictions from observed multimodal expressions and relative emotion ranks from local-global rankings for emotion recognition.

\section{B. Sequential Learning}

When temporal information is available, sequential learning can be applied to improve the accuracy of emotion recognition. Long Short Term Memory (LSTM) is often used for sequential learning due to its capability of modelling human memory [19], [20]. 


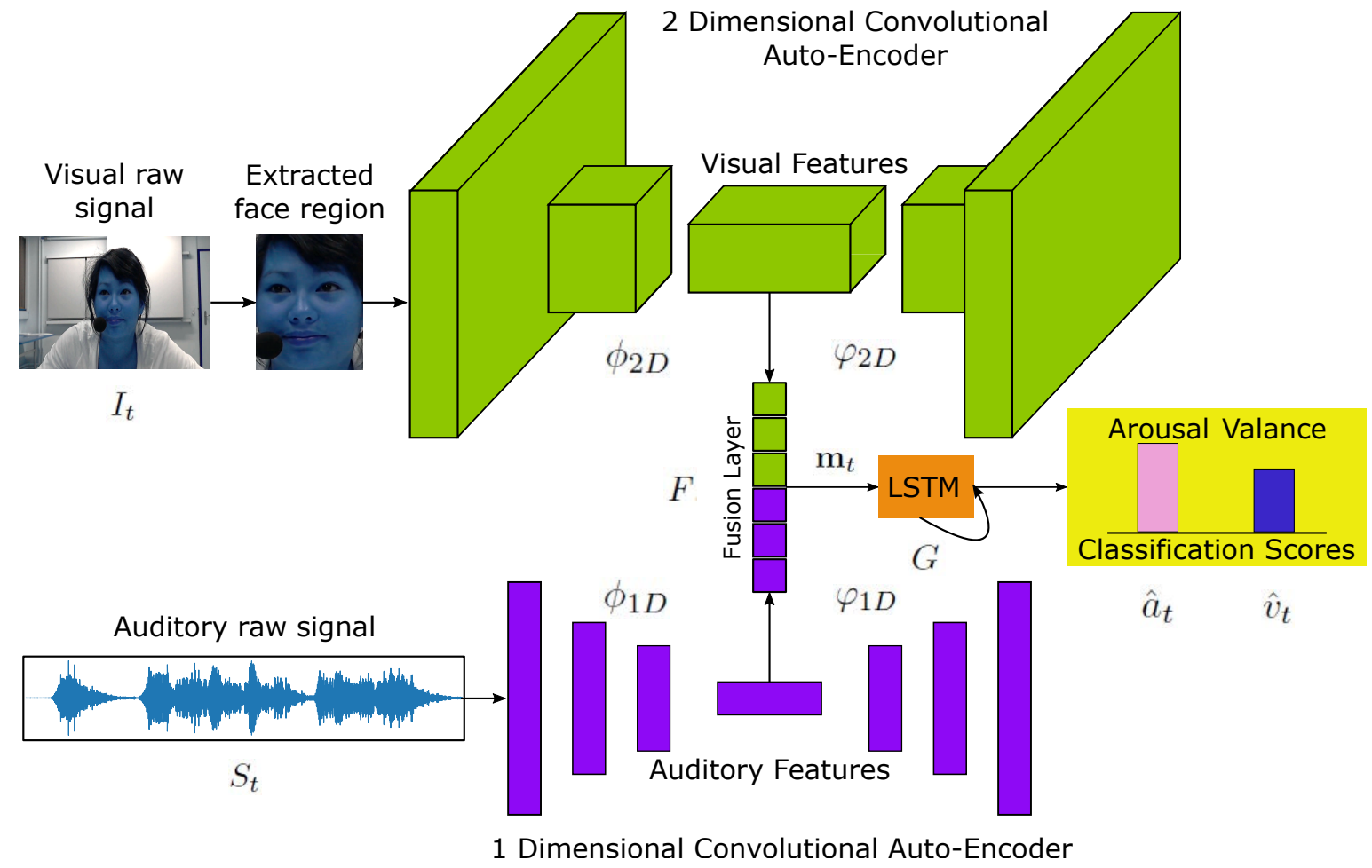

Fig. 2. Our proposed network architecture for multimodal dimensional emotion recognition.

Technically, LSTMs are recurrent neural networks (RNNs) integrated with some special gating architectures to control the access to memory cells [20]. These gates can also be used to prevent modifying the contents in the cells. LSTMs are, therefore, able to encode much longer range of patterns and propagate errors better than original recurrent neural networks [19]. Apart from being able to control the access to the contents in memory cells, the gates can also lean to target on specific parts of input sequences and refuse other parts. This feature allows LSTMs to be able to capture temporal information in sequential patterns.

Inspired by the advantages of LSTMs, many LSTM-based techniques have been developed for human emotion understanding from streaming data. For instance, Chen and Jin [21] and Wöollmer et al. [22] proposed LSTM-based networks for emotion recognition from data streams. Pei et al. [23] introduced a deep bidirectional LSTM-RNN, which was capable of modelling nonlinear relations in long-term history to handle both multimodal and unimodal emotion recognition tasks. In [3], [24], the authors developed an affective recognition system consisting of several deep neural networks to learn features at discrete times and an LSTM to model the temporal evolution of features overtime.

Despite recent promising achievements, current developments lack of ability to learn compact and representative features on individual domains and effectively learn multimodal features from multimodal data streams. To overcome these limitations, we propose to learn compact and representative features from individual domains using auto-encoders and fuse those domain-dependent features into multimodal features, integrated with temporal information using LSTM.

\section{Proposed Method}

In this section, we present an end-to-end system for recognition of dimensional emotion from multimodal data including visual and audio data streams.

\section{A. Data Pre-processing}

Input of our system is a pair of video and audio streams. For the video stream, we apply the Single Stage Headless (SSH) detector proposed in [25] to detect human faces in video frames. After that we resize the cropped faces to $96 \times 96$. The colour intensities in the cropped images are then normalised to $[-1,1]$.

For the audio stream, we segment the raw waveform signals of the stream into $0.2 \mathrm{~s}$ long sequences after normalising the time-sequences in order to obtain zero mean and unit variance. The normalisation aims at taking into account the variation in the loudness among different speakers. For a given input stream sampled at $16 \mathrm{kHz}$, each $0.2 \mathrm{~s}$ long sequence consists of 5 audio frames, each of which takes $0.04 \mathrm{~s}$ and is represented by a 640-dimensional vector. Note that each audio frame corresponds to a video frame in the input video stream.

\section{B. Network Architecture}

The proposed network architecture is illustrated in Fig. 2 Our architecture consists of two network branches, a 2D convolutional auto-encoder (2DConvAE) and a 1D convolutional autoencoder (1DConvAE), and a long short term memory (LSTM). Each network branch takes its corresponding input, e.g., the 2DConvAE receives input as an image while the 1DConvAE receives input as a $0.04 \mathrm{~s}$ audio frame. The latent layers of these 
TABLE I

DETAILED DESCRIPTION OF THE 2DCONVAE. CONV2D: 2D CONVOLUTIONAL LAYER. DECONV2D: 2D DECONVOLUTIONAL LAYER. FC: FULLY-CONNECTED LAYER.

\begin{tabular}{|c|c|c|c|c|}
\hline & Layer & Input size & $\begin{array}{l}\text { Filter size, Stride, } \\
\text { Out channels }\end{array}$ & Output size \\
\hline \multirow{3}{*}{ Encoder } & Conv2D & $96 \times 96 \times 3$ & {$\left[\begin{array}{c}1 \times 1,1,8 \\
3 \times 3,2,8 \\
1 \times 1,1,16\end{array}\right]$} & $48 \times 48 \times 16$ \\
\hline & Conv2D & $48 \times 48 \times 16$ & {$\left[\begin{array}{l}1 \times 1,1,16 \\
3 \times 3,2,16 \\
1 \times 1,1,32\end{array}\right]$} & $24 \times 24 \times 32$ \\
\hline & $\mathrm{FC}$ & 18432 & - & 2048 \\
\hline \multirow{2}{*}{ Decoder } & \multicolumn{2}{|c|}{ DeConv2D $24 \times 24 \times 32$} & {$\left[\begin{array}{l}1 \times 1,1,16 \\
3 \times 3,2,16 \\
1 \times 1,1,16\end{array}\right]$} & $48 \times 48 \times 16$ \\
\hline & \multicolumn{2}{|c|}{ DeConv2D $48 \times 48 \times 16$} & {$\left[\begin{array}{l}1 \times 1,1,8 \\
3 \times 3,2,8 \\
1 \times 1,1,3\end{array}\right]$} & $96 \times 96 \times 3$ \\
\hline
\end{tabular}

branches are fused into a multimodal representation, which is then fed to the LSTM for prediction of two dimensional emotion scores: arousal and valence.

Given an input video stream (of a speaker) including an image sequence and audio sequence, each image frame in the sequence is passed into the SSH detector [25] to detect the speaker's face. The face image is then fed to the 2DConvAE to learn visual features. Simultaneously, the corresponding audio frame is passed to the 1DConvAE to learn audio features. The features extracted from the latent layers of these auto-encoders are compact yet representative for their individual domains. Those features are then combined via a fusion layer before being fed to the LSTM for sequential learning of the features (for every $0.2 \mathrm{~s}$ long sequence) from input streams. The reason for the LSTM is to model the temporal variation of the audio and visual features that provides the contextual information of the input data.

The combination of auto-encoders and LSTM ensures that the learnt representations are compact, rich and complementary and thus make the architecture optimal and robust towards the recognition task from multimodal data streams.

1) $2 D$ ConvAE: follows the common practice of $2 \mathrm{D}$ autoencoders, e.g., [26]. The encoder of the 2DCovAE is composed of two residual blocks in the ResNet architecture [27], then enclosed by a fully-connected layer. These residual blocks play a central role in feature enrichment. The decoder of the 2DCovAE includes two residual blocks, which are designed by stacking six 2D de-convolutional layers. Leaky ReLU (LReLU) activation function is adopted after each convolutional layer and de-convolutional layer in our design. Table I provides a detailed description of the 2DConvAE branch while Fig. 3 visualises its architecture.

2) IDConvAE: realises an $1 \mathrm{D}$ auto-encoder applied to audio signal sequences. The encoder of the 1DConvAE is
TABLE II

DETAILED DESCRIPTION OF THE 1DCONVAE. CONV1D: 1D CONVOLUTIONAL LAYER, DECONV1D: 1D DECONVOLUTIONAL LAYER. FC: FULLY-CONNECTED LAYER.

\begin{tabular}{ccccc}
\hline Layer & Input size & $\begin{array}{c}\text { Filter size, Stride, } \\
\text { Out channels }\end{array}$ & $\begin{array}{c}\text { Output } \\
\text { size }\end{array}$ \\
\hline \multirow{2}{*}{ Encoder } & Conv1D & $1 \times 640 \times 1$ & {$[1 \times 20,1,40]$} & $1 \times 640 \times 40$ \\
\cline { 2 - 5 } & Maxpooling & $1 \times 640 \times 40$ & {$[1,2,1]$} & $1 \times 320 \times 40$ \\
\cline { 2 - 5 } & Conv1D & $1 \times 320 \times 40$ & {$[1 \times 40,1,40]$} & $1 \times 320 \times 40$ \\
\cline { 2 - 5 } & Maxpooling & $1 \times 320 \times 40$ & {$[1,10,1]$} & $1 \times 32 \times 40$ \\
\hline FC & 1280 & - & 640 \\
\hline Decoder & FC & 640 & - & 1280 \\
\cline { 2 - 5 } & DeConv1D & $1 \times 320 \times 4$ & {$[1 \times 20,1,40]$} & $1 \times 320 \times 40$ \\
\hline & Upsampling & $1 \times 320 \times 40$ & {$[1,2,1]$} & $1 \times 64 \times 40$ \\
\hline & DeConv1D & $1 \times 640 \times 40$ & {$[1 \times 40,1,1]$} & $1 \times 640 \times 1$ \\
\hline
\end{tabular}

an 1D convolutional neural network. We adopt the network architecture proposed by Tzirakis et al. [3] in our design for the encoder of the 1DConvAE. Specifically, the 1DConvAE's encoder includes two 1D convolutional layers, each of which is followed by a max pooling layer. Two fully-connected layers are subsequently attached. The decoder of the 1DConv-AE is formed by stacking one 1D de-convolutional layer, followed by one upsampling layer and then another 1D de-convolutional layer. We summarise the architecture of the 1DConvAE in Table III and Fig. 4

3) LSTM: has demonstrated a powerful capability of learning long-range contextual information in sequential patterns [28]. Motivated by this power, we adopt a 2-layer LSTM with 512 cells for each layer to model the temporal and contextual information in multimodal features learnt by the 2DConvAE and 1DConvAE. Readers are referred to [29] for more detail on LSTM implementation.

\section{Joint Learning}

Our goal is to learn compact and representative features commonly shared in both visual and auditory domain for prediction of dimensional emotion scores.

For the ease of presentation, we first introduce important notations used in our method. We denote the encoder and decoder of the 2DConvAE as $\phi_{2 D}$ and $\varphi_{2 D}$ respectively. Similarly, the encoder and decoder of the 1DConvAE are denoted as $\phi_{1 D}$ and $\varphi_{1 D}$ respectively. Let $F$ denote the fusion layer which concatenates the features learnt by $\phi_{2 D}$ and $\phi_{1 D}$. The LSTM is represented by $G$, receiving input from the fusion layer $F$. Let $I_{t}$ denote a facial image obtained by applying the SSH face detector on an input image frame at time step $t$. Let $S_{t}$ denote the corresponding sound segment of the facial image $I_{t}$. Given a pair of input $\left(I_{t}, S_{t}\right)$, let $a_{t}$ and $v_{t}$ be the ground truth arousal and valence respectively, and $\hat{a}_{t}$ and $\hat{v}_{t}$ be the predicted arousal and valence of the input $\left(I_{t}, S_{t}\right)$. The training procedure can be described as follows. 


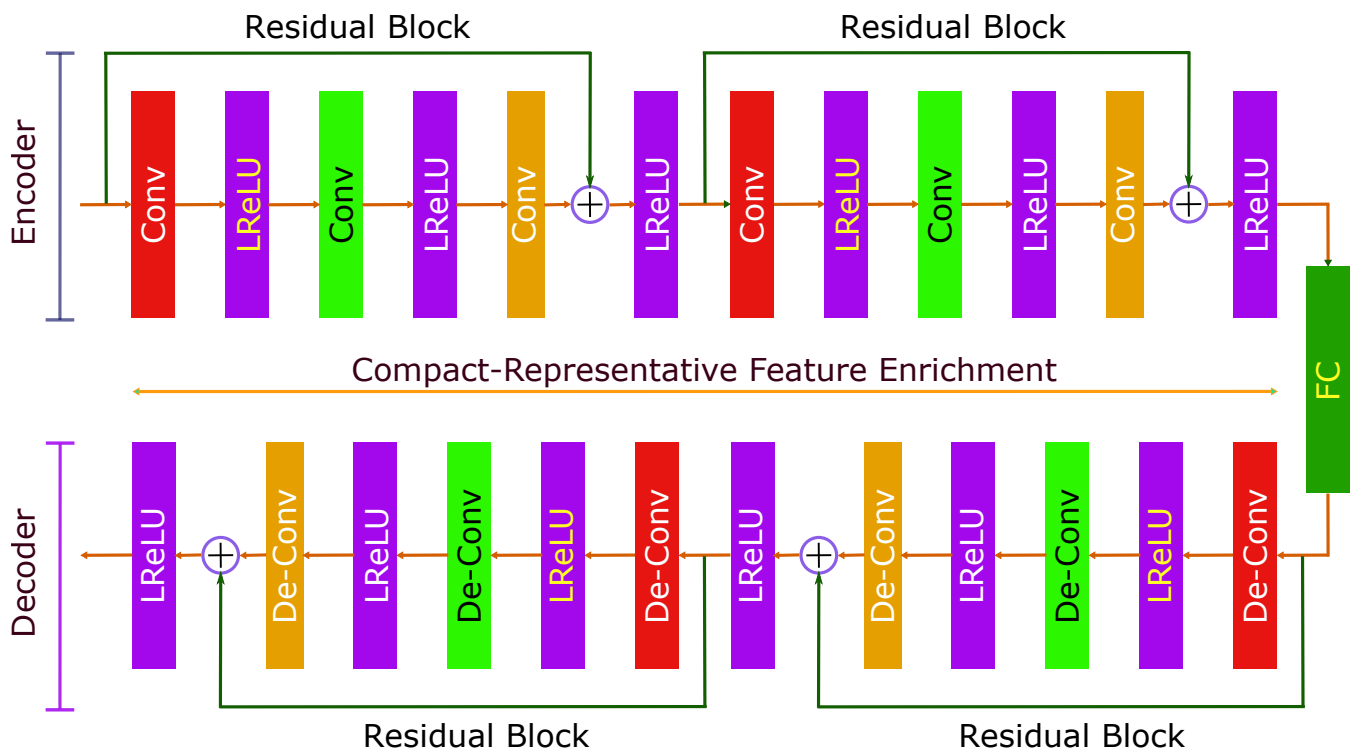

Fig. 3. Architecture of the 2DConvAE.

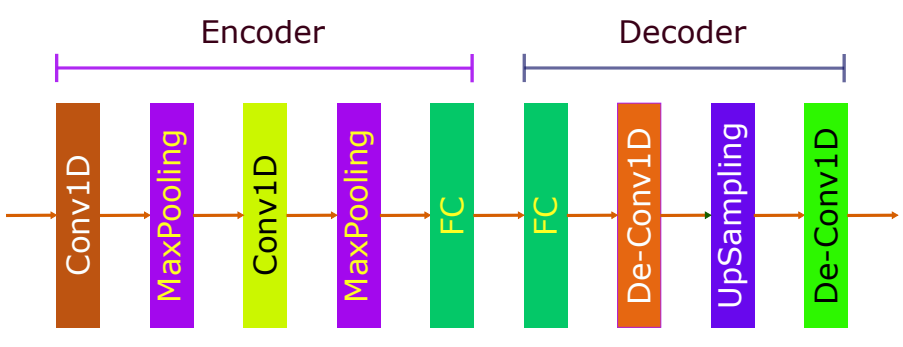

Compact-Representative Feature Enrichment

Fig. 4. Architecture of the 1DConvAE.

Given the input pair $\left(I_{t}, S_{t}\right)$, to learn compact and representative visual and audio features in individual domains, the auto-encoders 2DConvAE and 1DConvAE make use of the 2D and 1D encoders $\phi_{2 D}$ and $\phi_{1 D}$, and the 2D and 1D decoders $\varphi_{2 D}$ and $\varphi_{1 D}$, and result in an output pair of reconstructed image frame $\hat{I}_{t}$ and speech frame $\hat{S}_{t}$ where,

$$
\begin{gathered}
\hat{I}_{t}=\varphi_{2 D}\left(\phi_{2 D}\left(I_{t}\right)\right), \\
\hat{S}_{t}=\varphi_{1 D}\left(\phi_{1 D}\left(S_{t}\right)\right) .
\end{gathered}
$$

The quality of an auto-encoder can be measured via the similarity between an original signal and its reconstructed version after being processed through the auto-encoder. Autoencoders, thus, can ensure the representative quality of their encoded representations. In this work, we define the losses of our auto-encoders using $\ell_{2}$-norm as,

$$
\begin{aligned}
& \mathcal{L}_{2 D}=\sum_{t=1}^{n}\left\|\hat{I}_{t}-I_{t}\right\|_{2}^{2}, \\
& \mathcal{L}_{1 D}=\sum_{t=1}^{n}\left\|\hat{S}_{t}-S_{t}\right\|_{2}^{2}
\end{aligned}
$$

where $\hat{I}_{t}$ and $\hat{S}_{t}$ are defined in Eq. (1) and Eq. (2) respectively, $n$ is the number of samples (i.e., image/speech frames) in a current training batch.

To learn multimodal features, the fusion layer $F$ is used to fuse features extracted from the latent layers of the autoencoders. Specifically, the multimodal feature vector for the input pair $\left(I_{t}, S_{t}\right)$ is denoted as $\mathbf{m}_{t}$ and defined as,

$$
\mathbf{m}_{t}=F\left(\phi_{2 D}\left(I_{t}\right), \phi_{1 D}\left(S_{t}\right)\right)=\phi_{2 D}\left(I_{t}\right) \oplus \phi_{1 D}\left(S_{t}\right)
$$

where $\oplus$ represents a concatenating operator.

The multimodal feature vector $\mathbf{m}_{t}$ is then fed to the LSTM $G$ which estimates the arousal value $\hat{a}_{t}$ and valence value $\hat{v}_{t}$ of $\mathbf{m}_{t}$ based on its precedent observations, i.e.,

$$
\left(\hat{a}_{t}, \hat{v}_{t}\right)=G\left(\mathbf{m}_{t},\left\{\left(\mathbf{m}_{t-i}, \hat{a}_{t-i}, \hat{v}_{t-i}\right)\right\}_{i=1}^{k}\right)
$$

where $k$ is the number of precedent observations used to determine the arousal and valence value of the observation at time step $t$.

To measure the quality of arousal and valence prediction, we adopt the Concordance Correlation Coefficient (CCC) proposed in [30]. CCC has been widely used in for loss functions of dimensional emotion recognition systems [2]. It validates the agreement between two time series (e.g., predictions and their corresponding ground truth annotations) by scaling their correlation coefficient with their mean square difference. In this way, predictions that are well correlated with the annotations but shifted in the value are penalised proportionally to their deviations. CCC takes values in the range $[-1,1]$, where +1 denotes perfect concordance and -1 indicates perfect discordance. Applying the CCC, we define the loss for emotion recognition $\mathcal{L}_{R e c}$ as follows,

$$
\mathcal{L}_{\text {Rec }}=1-0.5 \cdot\left(\rho_{a}+\rho_{v}\right)
$$


where $\rho_{a}$ and $\rho_{v}$ are the CCC of the arousal and valence respectively, calculated on a current training batch. In particular, we define,

$$
\begin{aligned}
& \rho_{a}=\frac{2 \sigma_{\hat{a}, a}}{\sigma_{\hat{a}}^{2}+\sigma_{a}^{2}+\left(\mu_{\hat{a}}-\mu_{a}\right)^{2}} \\
& \rho_{v}=\frac{2 \sigma_{\hat{v}, v}}{\sigma_{\hat{v}}^{2}+\sigma_{v}^{2}+\left(\mu_{\hat{v}}-\mu_{v}\right)^{2}}
\end{aligned}
$$

where, for instance, $\sigma_{\hat{a}, a}$ is the covariance of the predictions $\hat{a}$ and ground truth annotations of arousal $a, \sigma_{\hat{a}}^{2}$ and $\sigma_{a}^{2}$ are the variance of $\hat{a}$ and $a$ respectively, and $\mu_{\hat{a}}$ and $\mu_{a}$ are the mean of $\hat{a}$ and $a$ respectively. Note that those statistics are calculated on a current training batch. Similar interpretations can be applied to $\rho_{v}$.

Finally, the loss of entire network is defined as,

$$
\mathcal{L}=\alpha \mathcal{L}_{2 D}+\beta \mathcal{L}_{1 D}+\gamma \mathcal{L}_{R e c}
$$

where $\mathcal{L}_{2 D}, \mathcal{L}_{1 D}$, and $\mathcal{L}_{R e c}$ is presented in Eq. (3), Eq. (4), and Eq. (7), $\alpha, \beta$, and $\gamma$ are weights used to control the influence of sub networks and set empirically.

As shown in Eq. (10), the individual auto-encoders and the LSTM are jointly trained. This scheme makes the features learnt through the entire network compact, representative, and complementary from different domains.

\section{EXPERIMENTS}

\section{A. Dataset}

We conducted our experiments on the REmote COLlaborative and Affective (RECOLA) dataset introduced by Ringeval et al. [31]. This dataset consists of spontaneous and natural emotions represented by continuous values of arousal and valence. The dataset has four modalities including electro-cardiogram, electro-dermal activity, audio, and visual modality. There are 46 French speaking subjects involved in recordings of 9.5 hours in total. The recordings are labelled for every 5 minutes by three male and three female French-speaking annotators. The dataset is balanced among various factors including mother tongue, age, and gender. The dataset includes a training set with 14 subjects and a test set with 15 subjects. Each subject is associated with a recording (including visual and audio signal). Each recording consists of 7,500 frames for each visual and audio channel, resulting in a total of 105,000 training samples and 112,500 test samples for each signal type. Both the training and test set are organised in subject independent manner. Fig. 5] illustrates an example in the RECOLA dataset.

The RECOLA dataset has been used as a benchmark in the Audio-Visual Emotion Challenge and Workshop (AVEC) in years 2015, 2016, and 2018. However, its competition set is not publicly available. Therefore, in this work, we run experiments only on the provided test set of the RECOLA dataset.

\section{B. Implementation Details}

Our emotion recognition system receives input as a multimodal data stream including an image channel and a speech channel. The data stream of each channel is segmented into frames which are then passed to the proposed network architecture for processing.

Given a pair $\left(I_{t}, S_{t}\right)$ including an image frame $I_{t}$ and its corresponding speech frame $S_{t}$ at some time step $t$, the image frame $I_{t}$ is passed to the visual network branch (2DConvAE) to extract 2,048 visual features via $\phi_{2 D}\left(I_{t}\right)$. Similarly, the speech frame $S_{t}$ is forwarded to the speech network branch (1DConvAE) to extract 1,280 auditory features via $\phi_{1 D}\left(S_{t}\right)$. These output features are concatenated to form a 3,328 dimensional multimodal representation $\mathbf{m}_{t}$ as defined in Eq. (5). This representation is fed to the 2-layer LSTM (with 512 cells per layer) to extract long-range contextual information from the data stream. The output of the LSTM is finally attached to a fully-connected layer to predict the arousal and valence for the input data at time step $t$.

To predict the arousal and valence for the input pair $\left(I_{t}, S_{t}\right)$, the LSTM takes into account the last four time steps of $t$, i.e., $k$ in Eq. (6) is set to 4 .

Our proposed architecture was trained end-to-end via optimising the joint loss $\mathcal{L}$ defined in Eq. (10) on the training set of the RECOLA database. In our implementation, we empirically set the parameters in Eq. (10) as, $\alpha=1, \beta=1$, and $\gamma=0.01$. This is just a general and dataset independent setting, where $\alpha$ and $\beta$ are set to the same value to make a balance between the audio and visual information, and $\gamma$ is set to a smaller value to fit with the range of $\mathcal{L}_{\text {Rec }}$. Adam optimiser (with default values) was adopted. Mini-batch size was set to 32 and learning rate was set to 0.0001 .

The number of training steps was set to 50,000. All experiments in this paper were implemented in TensorFlow and conducted on 10 computing nodes: $3780 \times 64$-bit Intel Xeon Cores. Our proposed model was trained within 45 hours and required $10,485,760 \mathrm{~KB}$ of memory usage. Fig. 6 shows the learning curve of our model.

\section{Evaluation Protocol}

As commonly used in evaluation of dimensional emotion recognition [2], we measure the Root Mean Square Error (RMSE) of predicted dimensional emotion scores against the ground truth values as follow,

$$
E_{a v}=\sqrt{\frac{1}{N} \sum_{i=1}^{N}\left(\hat{a}_{i}-a_{i}\right)^{2}+\left(\hat{v}_{i}-v_{i}\right)^{2}}
$$

where $\hat{a}$ and $a$ are the predicted arousal and its ground truth value respectively, and $\hat{v}$ and $v$ are the predicted valence and its ground truth value respectively, $N$ is the total number of frames in an input sequence.

To further investigate the prediction performance in detail, we also calculate the RMSE on each individual emotion dimension as,

$$
\begin{aligned}
& E_{a}=\sqrt{\frac{1}{N} \sum_{i=1}^{N}\left(\hat{a}_{i}-a_{i}\right)^{2}} \\
& E_{v}=\sqrt{\frac{1}{N} \sum_{i=1}^{N}\left(\hat{v}_{i}-v_{i}\right)^{2}}
\end{aligned}
$$




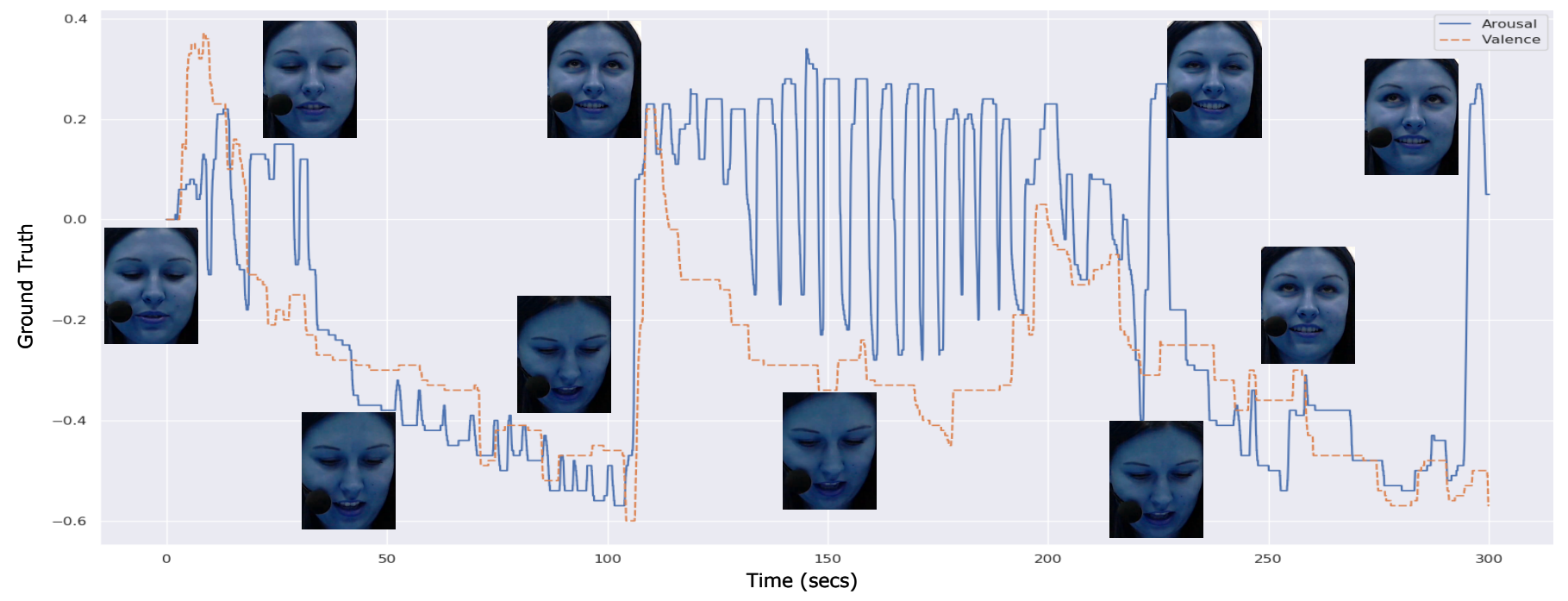

Fig. 5. Arousal and valance annotations over a part of a video in the RECOLA dataset. Corresponding frames are also illustrated. This figure shows the in-the-wild nature of the emotion data in RECOLA dataset (with different emotional states, rapid emotional changes, occlusions).

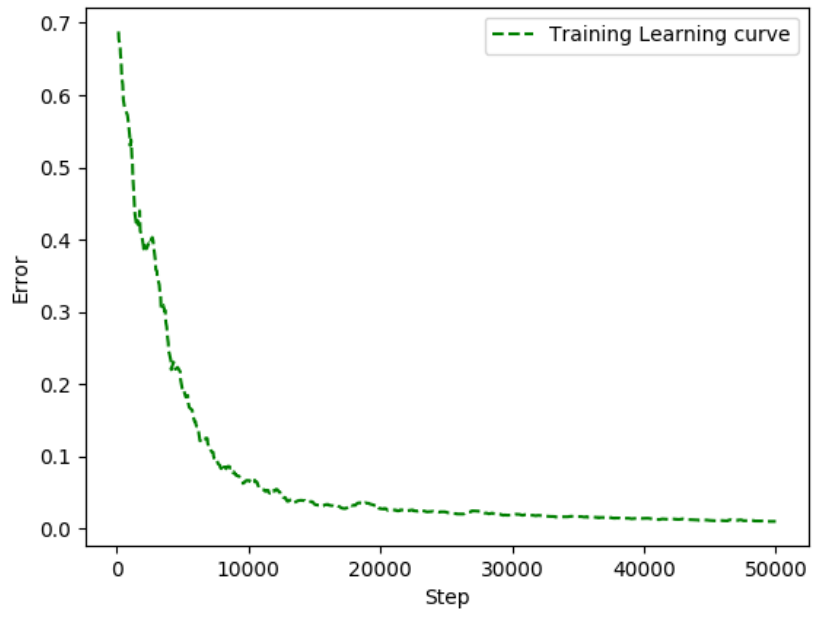

Fig. 6. Learning curve of our model.

The RMSE gives us an indication of how the derived emotion model is behaving, providing a direct comparative evaluation metric. Small values of the RMSE are desired.

We also use the CCC values, i.e., $\rho_{a}$ and $\rho_{v}$ defined in Eq. (8) and Eq. (9) respectively, as other metrics for emotion recognition performance. These metrics have also been used in recent affective computing studies, e.g., [32]. In contrast to RMSE, greater CCC values indicate better performance. As shown in our experiments, there is a discrepancy between the CCC and RMSE values, i.e., a better CCC value does not lead to a better RMSE value and vice versa. This is because CCC considers the variation of predicted values relatively to a test set and hence reflects the consistency of emotion recognition on that test set rather than the emotion recognition accuracy on individual samples. Meanwhile, RMSE is applicable to individual test samples, i.e., test cases even with a single test sample. Therefore, although both RMSE and CCC are employed in our experiments, RMSE could be considered as the metric for comparison of different models/architectures.

\section{Evaluation and Comparison}

Since there are several technical contributions proposed in this work, e.g., auto-encoders for learning multimodal features, LSTM for sequential learning of multimodal data streams, etc., we evaluate each of these contributions in our experiments. In addition, for each dimension in the evaluations, we also compare our method with existing related works.

1) Multimodal vs Unimodal: We first evaluated multimodal and unimodal approach in emotion recognition. Recall that our proposed multimodal architecture combines two unimodal branches, each of which focuses on a single domain (visual or audio domain). To make a comprehensive comparison between the muiltimodal and unimodal approaches, we respectively removed one of the two branches from our architecture and retrained the remaining ones from scratch to achieve unimodal architectures. For instance, the unimodal architecture for the visual part is denoted as "2DConvAE-LSTM" and obtained by taking off the speech network branch (see Fig. 2) while keeping the LSTM which receives input from only the latent layer of the 2DConvAE. Similar action was applied to the audio part to create "1DConvAE-LSTM."

Table [II] compares unimodal and multimodal approaches. As shown in the table, there is an inconsistency in the performance of the unimodal architectures. Specifically, the audio architecture (1DConvAE-LSTM) outperforms the visual one (2DConvAE-LSTM) in prediction of arousal while the visual architecture shows better performance than its counterpart in prediction of valence. Compared with these sub-models, our proposed multimodal architecture ("2DConvAE-1DConvAELSTM") combining both the unimodal architectures shows superior performance with lowest RMSE on prediction of both emotion dimensions.

We also compared our multimodal architecture with other two unimodal architectures proposed in [3] in Table III] Specifically, the authors in [3] combined CNNs and LSTM on visual and audio domain separately. As shown in Table IIII our multimodal architecture achieves lower RMSE than all 
TABLE III

COMPARISON OF MULTIMODAL AND UNIMODAL ARCHITECTURES. BEST PERFORMANCES ARE HIGHLIGHTED.

\begin{tabular}{|l||c|c|c|c|c|}
\hline \multicolumn{1}{|c||}{ Metric } & \multicolumn{3}{c|}{ RMSE } & \multicolumn{2}{c|}{ CCC } \\
\cline { 2 - 6 } Architecture & $E_{a}$ & $E_{v}$ & $E_{a v}$ & $\rho_{a}$ & $\rho_{v}$ \\
\hline 2DConvAE-LSTM & 0.538 & 0.214 & 0.579 & -0.010 & 0.036 \\
\hline 1DConvAE-LSTM & 0.493 & 0.237 & 0.547 & 0.185 & -0.003 \\
\hline 2DConv-LSTM in $[3]$ & 0.476 & 0.192 & 0.514 & 0.024 & 0.142 \\
\hline 1DConv-LSTM in $[\overline{3} \mid$ & 0.517 & 0.251 & 0.574 & 0.214 & 0.013 \\
\hline Our 2DConvAE-1DConvAE-LSTM & $\mathbf{0 . 4 7 4}$ & $\mathbf{0 . 1 8 7}$ & $\mathbf{0 . 5 1 0}$ & 0.102 & 0.155 \\
\hline
\end{tabular}

TABLE IV

EVALUATION OF AUTO-ENCODERS. BEST PERFORMANCES ARE HIGHLIGHTED.

\begin{tabular}{|l||c|c|c|c|c|}
\hline \multirow{2}{*}{ Metric } & \multicolumn{3}{c|}{ RMSE } & \multicolumn{2}{c|}{ CCC } \\
\cline { 2 - 6 } Architecture & $E_{a}$ & $E_{v}$ & $E_{a v}$ & $\rho_{a}$ & $\rho_{v}$ \\
\hline 2DConv-1DConvAE-LSTM & 0.506 & $\mathbf{0 . 1 8 4}$ & 0.539 & 0.007 & 0.149 \\
\hline VGG-1DConvAE-LSTM & 0.513 & 0.190 & 0.547 & 0.049 & 0.150 \\
\hline 2DConv-1DConv-LSTM 3 & 0.488 & $\mathbf{0 . 1 8 4}$ & 0.522 & 0.097 & 0.200 \\
\hline Our 2DConvAE-1DConvAE-LSTM & $\mathbf{0 . 4 7 4}$ & 0.187 & $\mathbf{0 . 5 1 0}$ & 0.102 & 0.155 \\
\hline
\end{tabular}

other unimodal ones on both arousal and valence prediction. However, the audio model in [3] achieves the highest CCC on arousal prediction while the visual model in [3] shows a dominant $\mathrm{CCC}$ on valence prediction.

2) Feature Learning with Auto-Encoders: In this experiment, we evaluated the contributions of the auto-encoders, i.e., the 2DConvAE and 1DConvAE, to the prediction performance of our architecture. First, to investigate the impact of the autoencoders on individual domains, we re-designed the unimodal branches by using only the encoders in those branches and measured their performances. As shown in Table III] the 1DConvAE (i.e., "1DConvAE-LSTM") improves the accuracy of the audio branch (i.e., "1DConv-LSTM") on both arousal and valence prediction. Specifically, the improvements are $4.6 \%$ on $E_{a}$ (from 0.517 to 0.493 ) and $5.6 \%$ on $E_{v}$ (from 0.251 to 0.237 ), leading to an overall improvement of $4.7 \%$ on $E_{a v}$ (from 0.574 to 0.547 ). In contrast, the 2DConvAE (i.e., "2DConvAE-LSTM") incurs a decrease of $11.5 \%$ on $E_{a}$ (from 0.476 to 0.538 ) and $10.3 \%$ on $E_{v}$ (from 0.192 to 0.214 ), and $11.2 \%$ on $E_{a v}$ (from 0.514 to 0.579 ), compared with "2DConv-LSTM". This decrease is probably because, compared with the $1 \mathrm{DConvAE}$, the $2 \mathrm{DC}$ ConvAE requires more training data since it contains more parameters (see Table I] and Table (II). Moreover, auto-encoders are beneficial to deal with noise which commonly occurs in auditory data. This explains the improvement gained by the auto-encoder in the audio branch. Meanwhile, facial images are cropped and hence excludes background noise. Unlike RMSE, Table III shows a consistent decrease of CCC values on both arousal and valence prediction using auto-encoders.

However, our experimental results show that the incorporation of both the 2DConvAE and 1DConvAE compensated the weakness of each individual component, achieving improved overall performance (see Table IV]. Since the autoencoder did not seem to bring benefit over its encoder part in 2D domain, we further investigated the decoder part of the $2 \mathrm{DConvAE}$ by removing it from the 2DConvAE while remaining other components of the entire architecture un- changed. We name this variant "2DConv-1DCovAE-LSTM", i.e., 2D convolutional encoder + 1DCovAE + LSTM. We also applied the well-known VGG architecture in [33] as an alternative for the 2D encoder and created a so-called "VGG-1DCovAE-LSTM" model. Like "2DConv-1DCovAELSTM", "VGG-1DCovAE-LSTM" does not include decoder part. We report the performance of all the variants in Table IV] The results in Table IV again confirm the combination of both the 2DConvAE and 1DConvAE to improving the overall performance of our architecture.

We compared our architecture with the one proposed in [3], which employed the same encoder (with our architecture) for the audio branch and the pre-trained ResNet in [27] for the visual branch. The same LSTM was used in both the architectures. Note that our 2D encoder is simpler (with less layers) than the one in [3] while decoders are not used in [3]. The method in [3] is currently the best model on the competition set of the RECOLA dataset in the AVEC. For fair comparison, we trained our model and [3] from scratch on the same training set and tested them on the test set of the RECOLA dataset.

We report the prediction performance of our model and the one in [3] in Table IV] As shown in our experimental results, compared with the network architecture proposed in [3], our model was slightly inferior in prediction of valence (see $E_{v}$ ) but significantly more prominent in prediction of arousal (see $E_{a}$ ), leading to a better overall performance (see $E_{a v}$ ). However, the model in [3] showed higher CCC values.

3) Sequential Learning with LSTM: LSTM has been widely exploited in dimensional emotion recognition from data streams. For instance, Kollias and Zafeiriou [24] proposed to use two LSTMs, each of which for one unimodal data stream (e.g., visual or audio stream). We denote this approach as "2DConv-1DConv-2SLSTM." Unlike [24], in our architecture, we use only one LSTM receiving input from a fusion layer and producing predicted values for arousal and valence. Note that auto-encoders were not employed in [24]. Therefore, to better study the effect of using one LSTM vs two stream LSTM, we 
TABLE V

EVALUATION OF LSTM. BEST PERFORMANCES ARE HIGHLIGHTED.

\begin{tabular}{|l||c|c|c|c|c|}
\hline \multicolumn{1}{|c|}{ Metric } & \multicolumn{3}{c|}{ RMSE } & \multicolumn{2}{c|}{ CCC } \\
\cline { 2 - 7 } Architecture & $E_{a}$ & $E_{v}$ & $E_{a v}$ & $\rho_{a}$ & $\rho_{v}$ \\
\hline 2DConv-1DConv-2SLSTM & 0.493 & $\mathbf{0 . 1 8 7}$ & 0.527 & 0.008 & 0.113 \\
\hline 2DConvAE-1DConvAE-2SLSTM & 0.508 & 0.190 & 0.542 & -0.008 & 0.128 \\
\hline Our 2DConvAE-1DConvAE-LSTM & $\mathbf{0 . 4 7 4}$ & $\mathbf{0 . 1 8 7}$ & $\mathbf{0 . 5 1 0}$ & 0.102 & 0.155 \\
\hline
\end{tabular}

TABLE VI

PREDICTION PERFORMANCE OF OUR ARCHITECTURE WHEN VARYING THE NUMBER OF HIDDEN NODES USED IN EACH LAYER OF THE LSTM.

\begin{tabular}{|l||c|c|c|c|c|}
\hline \multirow{2}{*}{\multicolumn{1}{|c|}{ Metric }} & \multicolumn{3}{c|}{ RMSE } & \multicolumn{2}{c|}{ CCC } \\
\cline { 2 - 6 } \#Hidden nodes & $E_{a}$ & $E_{v}$ & $E_{a v}$ & $\rho_{a}$ & $\rho_{v}$ \\
\hline 32 & 0.509 & 0.190 & 0.543 & 0.044 & 0.120 \\
\hline 64 & 0.540 & 0.195 & 0.574 & -0.032 & 0.106 \\
\hline 128 & 0.502 & 0.205 & 0.542 & 0.037 & 0.082 \\
\hline 256 & 0.496 & 0.184 & 0.529 & 0.038 & 0.108 \\
\hline 512 (current setting) & 0.474 & 0.187 & 0.510 & 0.102 & 0.155 \\
\hline
\end{tabular}

TABLE VII

PREDICTION PERFORMANCE OF OUR ARCHITECTURE WHEN VARYING THE NUMBER OF TRAINING SAMPLES

\begin{tabular}{|l||c|c|c|c|c|}
\hline \multirow{2}{*}{ \#Training samples } & \multicolumn{3}{c|}{ RMSE } & \multicolumn{2}{c|}{ CCC } \\
\cline { 2 - 6 } & $E_{a}$ & $E_{v}$ & $E_{a v}$ & $\rho_{a}$ & $\rho_{v}$ \\
\hline 26,250 & 0.533 & 0.190 & 0.566 & -0.066 & 0.180 \\
\hline 52,500 & 0.508 & 0.197 & 0.545 & -0.017 & 0.144 \\
\hline 78,750 & 0.505 & 0.184 & 0.538 & 0.000 & 0.145 \\
\hline 105,000 (entire training set) & 0.474 & 0.187 & 0.510 & 0.102 & 0.155 \\
\hline
\end{tabular}

TABLE VIII

PREDICTION PERFORMANCE OF OUR ARCHITECTURE UNDER DIFFERENT RECOGNITION LOSS FUNCTIONS.

\begin{tabular}{|l||c|c|c|c|c|}
\hline \multirow{2}{*}{ Recognition loss } & \multicolumn{3}{c|}{ RMSE } & \multicolumn{2}{c|}{ CCC } \\
\cline { 2 - 6 } & $E_{a}$ & $E_{v}$ & $E_{a v}$ & $\rho_{a}$ & $\rho_{v}$ \\
\hline MSE loss & 0.483 & 0.342 & 0.592 & -0.086 & -0.024 \\
\hline CCC loss (current setting) & 0.474 & 0.187 & 0.510 & 0.102 & 0.155 \\
\hline
\end{tabular}

applied the two stream LSTM in [24] to our architecture, to create a so-called "2DConvAE-1DConvAE-2SLSTM" variant.

Table $\mathrm{V}$ compares different approaches using LSTM for sequential learning in dimensional emotion recognition. As shown in the table, our architecture achieves the best performance among all models on both emotion dimensions.

4) Ablation Study:

a) LSTM Dimension: We studied the effect of the hidden nodes in the LSTM to the prediction performance of our model. Specifically, we investigated the prediction performance of our architecture with regard to various numbers of hidden nodes in each layer in the LSTM.

We report these results in Table VI In general, there is a fluctuation in the prediction performance when varying the number of hidden nodes in the LSTM. Although the best configuration for prediction of valence is the LSTM with 256 nodes in each hidden layer, our current setting with 512 nodes in each hidden layer shows better performance in prediction of arousal and also achieves the best overall performance.

b) Training Data Size: We experimented our architecture by varying the numbers of training samples. In particular, we trained our model using a quarter, a half, three quarters of the training dataset and the entire training set, while still maintaining subject independent setting.

We report the performance of our model under different numbers of training samples in Table VII. It is clearly to see that the model consistently improves its performance accordingly to the training data size.

c) Recognition Loss: To train our proposed architecture, we define the recognition loss $\mathcal{L}_{R e c}$ using the $\mathrm{CCC}$ of arousal and valence (see Eq. (7)). We refer this loss to as CCC loss. In this experiment, we compared the $\mathrm{CCC}$ loss with the mean square error (MSE) loss, i.e., by setting $\mathcal{L}_{R e c}$ to $E_{a v}$, which is commonly used for regression.

We present the performance of our architecture under these two recognition loss functions in Table VIII We observed that our current setting (i.e., the CCC loss) outperformed the MSE loss on both RMSE and CCC metrics.

d) Multimodal Data Fusion: In this work, we propose to incorporate visual and auditory features learnt by autoencoders on individual domains using concatenation. The concatenated features are then fed to the LSTM for sequential learning to predict the final emotion outcomes. Our multimodal data fusion mechanism is simple yet effective in practice. To prove this, we implemented the attention-based fusion 
TABLE IX

PREDICTION PERFORMANCE OF OUR ARCHITECTURE USING DIFFERENT FUSION METHODS.

\begin{tabular}{|l||c|c|c|c|c|}
\hline \multirow{2}{*}{ Metric } & \multicolumn{3}{c|}{ RMSE } & \multicolumn{2}{c|}{ CCC } \\
\cline { 2 - 7 } Fusion method & $E_{a}$ & $E_{v}$ & $E_{a v}$ & $\rho_{a}$ & $\rho_{v}$ \\
\hline Attention-based fusion & 0.578 & 0.230 & 0.623 & 0.019 & 0.149 \\
\hline Concatenation (current setting) & 0.474 & 0.187 & 0.510 & 0.102 & 0.155 \\
\hline
\end{tabular}

method used in [34], [35]. Specifically, attention weights were computed based on the strength of emotion in audio features, and then applied to multimodal feature fusion via a weighted sum of the multimodal features.

Experimental results (see Table IX) show that our simple concatenation-based fusion operator significantly outperformed the attention-based fusion method in [34] on both arousal and valence prediction.

\section{CONCLUSION}

This paper proposes a deep network architecture for end-toend dimensional emotion recognition from multimodal data streams. The proposed architecture incorporates auto-encoders for learning multimodal features from visual and audio domains, and LSTM for learning contextual and temporal information from streaming data. Our proposed architecture enables learning compact, representative, and complementary features from multimodal data source. We implemented various baseline models and conducted extensive experiments on the benchmark RECOLA dataset. Experimental results confirmed the contributions of our work and the superiority of our proposed architecture over existing ones.

In this paper, we extract visual features from human faces. However, the entire body of a person (in some specific pose) also conveys useful information (e.g., the body language) and thus could be an important indicator for the person's emotion. We consider the combination of human appearance and speech for emotion recognition in our future work.

\section{REFERENCES}

[1] P. P. Liang, A. Zadeh, and L.-P. Morency, "Multimodal local-global ranking fusion for emotion recognition," in ACM International Conference on Multimodal Interaction, 2018, pp. 472-476.

[2] D. Kollias, P. Tzirakis, M. A. Nicolaou, A. Papaioannou, G. Zhao, B. Schuller, I. Kotsia, and S. Zafeiriou, "Deep affect prediction in-thewild: Aff-wild database and challenge, deep architectures, and beyond," International Journal of Computer Vision, vol. 127, no. 6, pp. 907-929, 2019.

[3] P. Tzirakis, G. Trigeorgis, M. A. Nicolaou, B. W. Schuller, and S. Zafeiriou, "End-to-end multimodal emotion recognition using deep neural networks," IEEE Journal of Selected Topics in Signal Processing, vol. 11, no. 8, pp. 1301-1309, 2017.

[4] S. Zafeiriou, D. Kollias, M. A. Nicolaou, A. Papaioannou, G. Zhao, and I. Kotsia, "Aff-wild: Valence and arousal 'in-the-wild'challenge," in IEEE International Computer Vision and Pattern Recognition Workshops, 2017, pp. 1980-1987.

[5] D. Kollias, M. A. Nicolaou, I. Kotsia, G. Zhao, and S. Zafeiriou, "Recognition of affect in the wild using deep neural networks," in IEEE International Computer Vision and Pattern Recognition Workshops, 2017, pp. 1972-1979.

[6] D. Kollias and S. Zafeiriou, "Expression, affect, action unit recognition: Aff-wild2, multi-task learning and arcface," in British Machine Vision Conference, 2019, pp. 1-15.

[7] — "Aff-wild2: Extending the aff-wild database for affect recognition," arXiv:1811.07770, 2018.
[8] —, "A multi-task learning \& generation framework: Valence-arousal, action units \& primary expressions," arXiv:1811.07771, 2018

[9] S. Zhang, S. Zhang, T. Huang, W. Gao, and Q. Tian, "Learning affective features with a hybrid deep model for audio-visual emotion recognition," IEEE Transactions on Circuits and Systems for Video Technology, vol. 8, no. 10, pp. 3030-3043, 2018.

[10] O. Russakovsky, J. Deng, H. Su, J. Krause, S. Satheesh, S. Ma, Z. Huang, A. Karpathy, A. Khosla, M. Bernstein, A. C. Berg, and L. FeiFei, "Imagenet large scale visual recognition challenge," International Journal of Computer Vision, vol. 115, no. 3, pp. 211-252, 2015.

[11] D. Tran, L. Bourdev, R. Fergus, L. Torresani, and M. Paluri, "Learning spatiotemporal features with $3 \mathrm{~d}$ convolutional networks," in IEEE International Conference on Computer Vision, 2015, pp. 4489-4497.

[12] P. Barros, C. Weber, and S. Wermter, "Emotional expression recognition with a cross-channel convolutional neural network for human-robot interaction," in IEEE-RAS International Conference on Humanoid Robots, 2015, pp. 582-587.

[13] P. Barros and S. Wermter, "Developing crossmodal expression recognition based on a deep neural model," Adaptive Behavior - Animals, Animats, Software Agents, Robots, Adaptive Systems, vol. 24, no. 5, pp. 373-396, 2016.

[14] H. Li, J. Sun, Z. Xu, and L. Chen, "Multimodal 2d+3d facial expression recognition with deep fusion convolutional neural network," IEEE Transactions on Multimedia, vol. 19, no. 12, pp. 2816-2831, 2017.

[15] Z. Zhang, J. Han, E. Coutinho, and B. Schuller, "Dynamic difficulty awareness training for continuous emotion prediction," IEEE Transactions on Multimedia, vol. 21, no. 5, pp. 1289-1301, 2019.

[16] W. Zheng, W. Liu, Y. Lu, B. Lu, and A. Cichocki, "Emotionmeter: A multimodal framework for recognizing human emotions," IEEE Transactions on Cybernetics, vol. 49, no. 3, pp. 1110-1122, 2019.

[17] J. Ngiam, A. Khosla, M. Kim, J. Nam, H. Lee, and A. Y. Ng, "Multimodal deep learning," in International Conference on International Conference on Machine Learning, 2011, pp. 689-696.

[18] W. Zhang, X. He, and W. Lu, "Exploring discriminative representations for image emotion recognition with cnns," IEEE Transactions on Multimedia, vol. 22, no. 2, pp. 515-523, 2020.

[19] N. Kalchbrenner, I. Danihelka, and A. Graves, "Grid long short-term memory," arXiv:1507.01526, 2015.

[20] S. Hochreiter and J. Schmidhuber, "Long short-term memory," Neural Computation, vol. 9, no. 8, p. 1735-1780, 1997.

[21] S. Chen and Q. Jin, "Multi-modal dimensional emotion recognition using recurrent neural networks," in International Workshop on Audio/Visual Emotion Challenge, 2015, pp. 49-56.

[22] M. Wöllmer, M. Kaiser, F. Eyben, B. Schuller, and G. Rigoll, "LSTMModeling of continuous emotions in an audiovisual affect recognition framework," Image and Vision Computing, vol. 31, no. 2, pp. 153-163, 2013.

[23] E. Pei, L. Yang, D. Jiang, and H. Sahli, "Multimodal dimensional affect recognition using deep bidirectional long short-term memory recurrent neural networks," in IEEE International Conference on Affective Computing and Intelligent Interaction, 2015, pp. 208-214.

[24] D. Kollias and S. Zafeiriou, "Exploiting multi-cnn features in cnn-rnn based dimensional emotion recognition on the omg in-the-wild dataset," arXiv:1910.01417, 2019.

[25] M. Najibi, P. Samangouei, R. Chellappa, and L. Davis, "SSH: Single stage headless face detector," in IEEE International Conference on Computer Vision, 2017, pp. 4875-4884.

[26] X. Mao, C. Shen, and Y.-B. Yang, "Image restoration using very deep convolutional encoder-decoder networks with symmetric skip connections," in Advances in Neural Information Processing Systems, 2016, pp. $2802-2810$

[27] K. He, X. Zhang, S. Ren, and J. Sun, "Deep residual learning for image recognition," in IEEE International Conference on Computer Vision and Pattern Recognition, 2016, pp. 770-778.

[28] Z. Zhang, F. Ringeval, J. Han, J. Deng, E. Marchi, and B. Schuller, "Facing realism in spontaneous emotion recognition from speech: Feature 
enhancement by autoencoder with lstm neural networks," in Interspeech, 2016, pp. 3593-3597.

[29] A. Graves, Supervised Sequence Labelling with Recurrent Neural Networks, ser. Studies in Computational Intelligence. Springer, 2012, vol. 385.

[30] L. I.-K. Lin, "A concordance correlation coefficient to evaluate reproducibility," Biometrics, vol. 45, no. 1, pp. 255-268, 1989.

[31] F. Ringeval, A. Sonderegger, J. Sauer, and D. Lalanne, "Introducing the recola multimodal corpus of remote collaborative and affective interactions," in IEEE International Conference on Automatic Face and Gesture Recognition, 2013, pp. 1-8.

[32] D. Ong, Z. Wu, Z. Tan, M. Reddan, I. Kahhale, A. Mattek, and J. Zaki, "Modeling emotion in complex stories: the stanford emotional narratives dataset," IEEE Transactions on Affective Computing, pp. 1-1, 2019.

[33] K. Simonyan and A. Zisserman, "Very deep convolutional networks for large-scale image recognition," in International Conference on Learning Representations, 2015, pp. 1-14.

[34] C. Hori, T. Hori, T.-Y. Lee, Z. Zhang, B. Harsham, J. R. Hershey, T. K. Marks, and K. Sumi, "Attention-based multimodal fusion for video description," in IEEE International Conference on Computer Vision, 2017, pp. 4193-4202.

[35] S. Yoon, S. Byun, and K. Jung, "Multimodal speech emotion recognition using audio and text," in IEEE Spoken Language Technology Workshop, 2018, pp. 112-118.

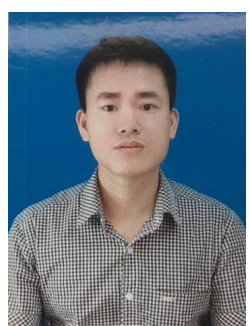

Dung Nguyen received the B.Eng. and M.Eng. degrees from the People Securiry Academy and the Vietnam National University - University of Enginerring and Technology in 2006 and 2013, respectively. He received his $\mathrm{PhD}$ degree in the area of multimodal emotion recognition using deep learning techniques from Queensland University of Technology in Brisbane, Australia in 2019. He is currently working as a research fellow at Monash University. His current research interests include computer vision, machine learning, multimodal deep learning, multimodal data processing, and affective computing.

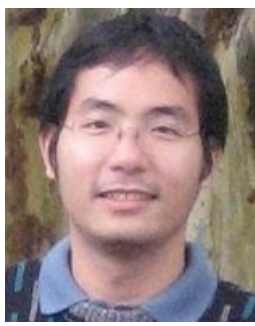

Duc Thanh Nguyen was awarded a $\mathrm{PhD}$ in Computer Science from the University of Wollongong, Australia in 2012. Dr Nguyen is currently a Senior Lecturer in the School of Information Technology, Deakin University, Australia. His research interests include computer vision and pattern recognition. He has published his work in highly ranked publication venues in Computer Vision and Pattern Recognition such as Pattern Recognition journal, CVPR, ICCV, ECCV, AAAI. Dr Nguyen is an area chair for the subject area "Multimedia Analysis and Understanding" of the IEEE International Conference on Multimedia and Expo (ICME) 2021 and has continuously severed as a technical program committee member for many premium conferences such as CVPR, ICCV, ECCV, AAAI, ICIP, PAKDD, SIGGRAPH, SIGGRAPHAsia. He has also been a reviewer for Pattern Recognition journal, IEEE Trans. Intell. Transp. Syst., IEEE Trans. Image Process., IEEE Trans. Multimedia, IEEE Trans. Circuits and Systems for Video Technology, IEEE Signal Processing Letters, Pattern Recognition, Image and Vision Computing, Scientific Reports/Nature.

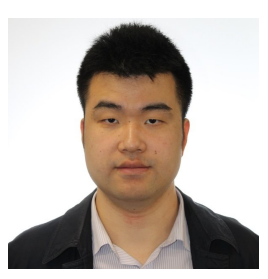

Rui Zeng received the B.S. degree in Computer Science from Wuhan Institute of Technology in 2012, the M.S. degree in electronic telecommunications in University of Rennes 1, the M.Eng. degree in Computer Science from Southeast University in 2015, and the $\mathrm{PhD}$ degree in the area of homography estimation from Queensland University of Technology in 2020. He was a research fellow in Australian Centre for Robotic Vision and eResearch Centre at Monash University. Dr Zeng is currently a postdoctoral research associate in the Brain and Mind Centre at the University of Sydney, Australia. His research interests include multi-view geometry, deep learning, and medical imaging.

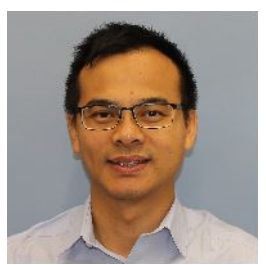

Thanh Thi Nguyen was a Visiting Scholar with the Computer Science Department at Stanford University, California, USA in 2015 and the Edge Computing Lab, John A. Paulson School of Engineering and Applied Sciences, Harvard University, Massachusetts, USA in 2019. He received an Alfred Deakin Postdoctoral Research Fellowship in 2016, a European-Pacific Partnership for ICT Expert Exchange Program Award from European Commission in 2018, and an Australia-India Strategic Research Fund Early- and Mid-Career Fellowship Awarded by The Australian Academy of Science in 2020. Dr Nguyen obtained a PhD in Mathematics and Statistics from Monash University, Australia in 2013 and has expertise in various areas, including artificial intelligence, deep learning, deep reinforcement learning, cyber security, IoT, and data science. He is currently a Senior Lecturer in the School of Information Technology, Deakin University, Victoria, Australia.

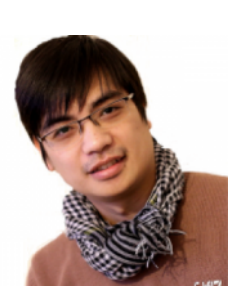

Son N. Tran is a Lecturer at the University of Tasmania. Son was a post-doctoral research fellow at the Commonwealth Scientific and Industrial Research Organisation, Australia. He holds a Ph.D. in Computer Science (2016) from City, University of London, an EU Erasmus Mundus joint MSc in Networks and e-Business Computing, University of Reading, and a BSc and MSc in Electronic Engineering from the Hanoi University of Technology. Tran is the recipient of the Erasmus Mundus certificate of achievement 2010 in recognition of an outstanding academic performance. His current research interest is learning and reasoning with visual objects.

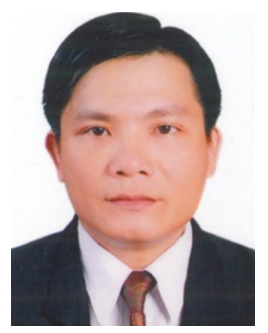

Thin Nguyen is a Senior Research Fellow with the Applied Artificial Intelligence Institute (A2I2), Deakin University, Australia. He graduated with a $\mathrm{PhD}$ in Computer Science from Curtin University, Australia in 2012 in the area of machine learning and social media analytics. His current research topic is strongly inter-disciplinary, bridging largescale data analytics, pattern recognition, genetics and medicine. His research direction is to utilize artificial intelligence methods to discover functional connections between drugs, genes and diseases. The domain of application emphasizes on personalized medicine, where he has developed personalized annotation-based networks for the prediction of breast cancer relapse. In that line of work, he won the first prize in the DREAM Single Cell Transcriptomics Challenge, organized by IBM Research \& Sage Bionetworks. 


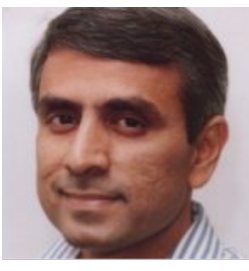

Sridha Sridharan has a BSc (Electrical Engineering) degree and obtained a MSc (Communication Engineering) degree from the University of Manchester UK and a $\mathrm{PhD}$ degree in the area of Signal Processing from Un iversity of New South Wales, Australia. He is a Life Senior Member of the Institute of Electrical and Electronic Engineers - IEEE (USA). He is currently with the Queensland University of Technology (QUT) where he is a Professor in the School Electrical Engineering and Robotics. Professor Sridharan is the Leader of the Research Program in Speech, Audio, Image and Video Technology (SAIVT) at QUT. He has published over 500 papers consisting of publications in journals and in refereed international conferences in the areas of Image and Speech technologies. He has graduated over $80 \mathrm{PhD}$ students at QUT. Prof Sridharan has also received a number of research grants from various funding bodies including commonwealth competitive funding schemes such as the Australian Research Council (ARC) and Cooperative Research Centres (CRC). Several of his research outcomes have been commercialised.

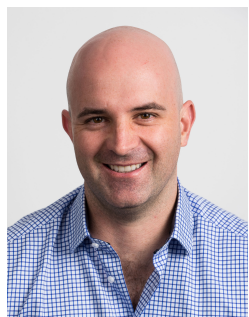

Clinton Fookes is a Professor in Vision \& Signal Processing at the Queensland University of Technology. He holds a BEng (Aerospace/Avionics), an $\mathrm{MBA}$, and a $\mathrm{PhD}$ in computer vision. He actively researches across computer vision, machine learning, signal processing and pattern recognition areas. He serves on the editorial boards for the Pattern Recognition Journal and the IEEE Transactions on Information Forensics \& Security. He is a Senior Member of the IEEE, an Australian Institute of Policy and Science Young Tall Poppy, an Australian Museum Eureka Prize winner, and a Senior Fulbright Scholar. 